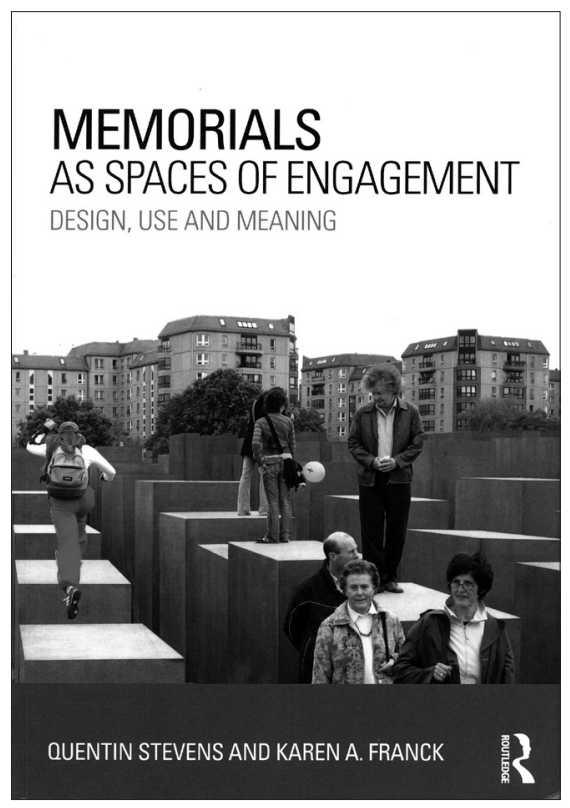

FIGURA 1. Portada del libro Memorials as Spaces of Engagement: Design, Use and Meaning, Quentin Stevens y Karen A. Franck, Londres/Nueva York, Routledge, 2016.

\section{Conmemorar lo indecible: museos y monumentos. Memorials as Spaces of Engagement: Design, Use and Meaning}

Commemorating the Unspeakable: Museums and Memorials. Memorials as Spaces of Engagement: Design, Use and Meaning

\section{Michael Andrés Forero Parra}

School of Museum Studies, University of Leicester (UoL, Reino Unido)

arkforero@yahoo.com

\section{Resumen}

Publicado por Routledge en 2016, escrito por los profesores Quentin Stevens (Instituto Real de Tecnología de Melbourne [RMIT, Royal Melbourne Institute of Technology], Australia) y Karen A. Franck (Instituto de Tecnología de Nueva Jersey [NJIT, New Jersey Institute of Technology], Estados Unidos de América), Memorials as Spaces of Engagement: Design, Use and Meaning reúne más de 10 años de experiencia en el análisis de monumentos conmemorativos y sus relaciones con el espacio público en diversas ciudades alrededor del mundo. Su contenido, por lo tanto, resultará muy atractivo para arquitectos, urbanistas, museólogos e interesados en estudiar contenidos sobre memoria, patrimonio y duelo. Suficientemente ilustrada, y con una mirada transversal, la obra aborda diversos temas, como el diseño, la interacción y la administración de monumentos, que tienen eco con un género de museos conmemorativos que en los años recientes se han establecido en capitales de América Latina como Buenos Aires (Argentina), Santiago (Chile), Lima (Perú), la Ciudad de México (México) y Bogotá (Colombia). Es un importante volumen, y un ejemplo fehaciente de que el patrimonio y la memoria tienen un futuro que recordar.

\section{Palabras clave}

monumentos conmemorativos; museos de la memoria; conflicto armado y patrimonio; América Latina

\section{Abstract}

Published by Routledge (2016) and written by professors Quentin Stevens (Royal Melbourne Institute of Technology [RMIT], Australia) and Karen Franck (New Jersey Institute of Technology [NIIT,], United States of America), Memorials as Spaces of Engagement: Design, Use, and Meaning collects more than ten-year experience analysing memorials and their relationships with public space through different cities worldwide. Hence its content will be of great interest to architects, urbanism specialists, museum professionals, and those studying memory, heritage and, mourning. With generous comprehension and through a transversal approach, this book examines diverse the- 
mes such as memorial's design, interaction, and management, as well as issues related to commemorative museums, a genre that have been established within the last years in Latin American capitals, such as Buenos Aires (Argentina), Santiago (Chile), Lima (Peru), Mexico City (México), and Bogota (Colombia). This is a remarkable volume, which exemplifies that both heritage and memory have a future to remember.

\section{Key Words}

commemorative memorials; memory museums; armed conflict and heritage; Latin America

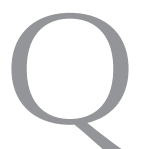

uentin Stevens, profesor asociado de diseño urbano del Royal Melbourne Institute of Technology (RMIT [Instituto Real de Tecnología de Melbourne], Australia), y Karen A. Franck, profesora del Departamento de Arquitectura y Diseño del New Jersey Institute of Technology (NIIT [Instituto de Tecnología de New Jersey], Estados Unidos de América) recogen en Memorials as Spaces of Engagement: Design, Use and Meaning, _publicado por Routledge en 2016,- más de 10 años de experiencia en el análisis in situ de monumentos conmemorativos y sus relaciones con el espacio público en ciudades emblemáticas, tales como Washington (EUA), Berlín (Alemania), Londres (Reino Unido), Melbourne (Australia) y Ottawa (Canadá). En efecto, el Vietnam Veterans Memorial (Monumento a los Veteranos de Vietnam, Washington, EUA), diseñado por la arquitecta Maya Lin, el NAMES Project (Proyecto NAMES) para el AIDS Memorial Quilt (Edredón Conmemorativo sobre el SIDA, Washington, EUA), concebido por el activista Cleve Jones, y el Denkmal für die Ermordeten Juden Europas (Monumento a los Judíos de Europa Asesinados, Berlín, Alemania), creado por el arquitecto Peter Eisenman, son tres ejemplos paradigmáticos referidos en el libro en reseña que evidencian notables transformaciones en el diseño de memoriales desde hace un par de décadas.
Por otra parte, aunque el volumen sealeja explícitamente del campo museológico y concibe al museo como "una estrategia suplementaria de ciertos monumentos alrededor del mundo" (Stevens y Franck 2016: 184; cursivas del autor), propone cinco ideas fundamentales que, como demostraré a continuación, ciertamente se corresponden con un género de museos conmemorativos creados en los años recientes en varias capitales de América Latina: Buenos Aires (Argentina), Santiago (Chile), Lima (Perú), la Ciudad de México (México) y Bogotá (Colombia).

Vale señalar que los museos de memoria y derechos humanos consagrados al reconocimiento de las víctimas de dictaduras, genocidios, actos terroristas, entre otros, con frecuencia se han concebido como consecuencia de recomendaciones de comisiones de la verdad o de actos legislativos de reparación simbólica, en contextos ya de reconciliación, ya de posconflicto, ya de construcción de paz (Carter 2015:208). Precisamente, una de las primeras ideas de Memorials as Spaces of Engagement: Design, Use and Meaning es que el desplazamiento de conmemoraciones enaltecedoras del triunfo, la victoria y la grandeza de un único vencedor han conducido al reconocimiento del sacrificio, el trauma y el esfuerzo de personas de distinta orientación sexual, condición de discapacidad, origen étnico o posición socioeconómica (Stevens y Franck 2016:35). En efecto, para Stevens y Franck (2016:35), estos nuevos protagonistas de la historia han desafiado a los diseñadores y gestores de memoriales, quienes han optado alternativamente por diversas aproximaciones para su representación; por ejemplo, se ha trascendido de aquellas grandes estatuas para individuos distinguidos a las inserciones arquitectónicas que en espacios públicos inscriben nombres sin distinción de rango, clase o nacionalidad.

Reflexiones en torno del relato grandilocuente, la figura masculina del héroe o la adición de memoriales que complementan a otro resuenan en el ámbito de los museos de memoria y, a la vez, cuestionan diversas instancias: el lugar que ocupan los perpetradores en la narración curatorial, las maneras en que se estimulan la participación y el diálogo en el interior del museo o, en el caso del Museo Memoria y Tolerancia (Ммут, México), de la Ciudad de México, las formas en que la institución incorpora la historia nacional en un guión museográfico que "perdió la oportunidad de crear un sitio que podría haber transformado su paisaje y sus significados" (Bergdoll 2011:27; cursivas del autor).

Desde mi punto de vista, dichos cambios en la narración de la historia, aunados a la incorporación de nuevas perspectivas, parecen no sólo haber construido nuevas formas de procesar el sufrimiento sino también proporcionaron cambios culturales ante el dolor y la muerte, reflejados en acciones e iniciativas "que conmemoran y dignifican la memoria de las víctimas y sensibilizan a la sociedad civil" (GMH 2013:387). Así, aunque el enfoque del libro infortunadamente no emprende una reflexión directa sobre el significado del duelo en nuestra contemporaneidad, incluye en su análisis lo que el propio texto ha denominado memoriales informales (Stevens y Franck 2016:4), los cuales aluden a acciones creadas en el espacio público tras una muerte repentina y sin una sanción oficial o una dirección preestablecida.

Acerca de esta segunda idea, Stevens y Franck (2016:4) redundan en que tales prácticas empáticas y afectivas invocan, a su vez, ciertos usos y actividades no esperados en sitios de conmemoración, tales como la danza, la comida o el canto, como lo advertí en una reciente visita al Parque de la Memoria Monumento a las Víctimas del Terrorismo de Estado, de Buenos Aires (Argentina), espacio público de 14 ha de extensión, a orillas del Río de la Plata, constituido en un "testimonio mudo del destino de muchas de las víctimas" (PDM 2014), 
donde se reúnen, además de los interesados en el arte contemporáneo o los familiares de desaparecidos, quienes practican jogging o se sientan en el prado alrededor de un picnic, lo cual necesariamente entabla relaciones distintas con la historia, la memoria y el arte.

A la par, nuevos proyectos de memoria que suscitan aproximaciones diferentes al patrimonio del conflicto armado se han creado, por ejemplo, por medio de la música. Tal es el caso de Tocó cantar. Travesía contra el olvido (CNMH 2015), del Museo Nacional de la Memoria de Colombia, proyecto del Centro Nacional de Memoria Histórica ${ }^{1}$ y de Música x Memoria (MMDH 2011), del Museo de la Memoria y los Derechos Humanos de Chile. ${ }^{2}$

En ese orden de ideas, los monumentos conmemorativos se han convertido en "escenarios teatrales" así para encuentros imprevistos como para los previamente organizados (Stevens y Franck 2016:126), analogía ya expuesta en otros textos referidos a museos de memoria (Williams 2007:96), a teorías feministas en museos (Hein 2010:60) y a estéticas teatrales en diseños expositivos (Crawley 2012:12). A partir de esta tercera idea

1 El Centro Nacional de Memoria Histórica (Colombia), es un establecimiento público del orden nacional, creado por la Ley de Víctimas y Restitución de Tierras (1448 de 2011), que tiene como uno de sus objetivos estratégicos diseñar, construir y entregar al país un Museo de la Memoria como un espacio de dignificación de las víctimas y de promoción de una cultura respetuosa de los derechos humanos (CNMH 2016).

2 El Museo de la Memoria y los Derechos Humanos (Chile), fue inaugurado el 11 de enero de 2010. Su origen se encuentra en las recomendaciones de la Comisión de Verdad y Reconciliación, Informe Rettig, en las políticas de apoyo a la construcción de memoriales del presidente Ricardo Lagos ("No hay mañana sin ayer") y en la decisión de la presidenta Michelle Bachelet de dar respuesta a las demandas de las organizaciones de familiares y de organismos de defensa de los derechos humanos, cuyos archivos fueron declarados "Memoria del Mundo" por la UNESCO (MMDH 2017). se puede hablar, entonces, de una performance museográfica, entendida como una serie de experiencias sensoriales que sugieren hallar significado mediante el cuerpo, que, a su vez, afecta y se afecta tanto por otros visitantes como por el espacio (Mac Leod, et al. 2012:XIX). Así, en el Sitio de Memoria ESMA (Escuela de Mecánica de la Armada), ${ }^{3}$ en Buenos Aires (Argentina), una serie de proyecciones envuelve a los visitantes al inicio de su recorrido; en ella se presentan simultáneamente imágenes, datos, narraciones y sonidos, con lo que se marcan el sentido y el propósito del lugar donde sucedieron hechos victimizantes realizados durante la dictadura militar argentina entre los años 1976 y 1983 (EMDH 2011b). Este recurso cinematográfico inmersivo también está presente, en una mayor medida — como se puede evidenciar en su visita - en el Imperial War Museum de Mánchester (IWM, [Museo Imperial de Guerra], Reino Unido), que utiliza todo el espacio de las salas de exposición para transformarse en una atmósfera teatral. Asimismo, aunque menos protagónicamente, el mismo recurso se utiliza en la Dauerausstellung im Ort der Information (Exposición permanente en el Centro de Información del Memorial a los Judíos de Europa Asesinados, Berlín, Alemania), sobre cuyo piso y paredes se proyectan sucesiva y cambiantemente los nombres de las víctimas del Holocausto y los lugares donde se las ejecutó (FMMJE 2010:23).

Para Stevens y Franck (2016:29), esta serie de experiencias soportadas

${ }^{3}$ La Escuela de Mecánica de la Armada (ESMA), fundada en 1924 durante la presidencia de Marcelo T. de Alvear, se utilizó como centro de instrucción militar que incluía: la Escuela de Mecánica, la Escuela de Guerra Naval y el Casino de Oficiales. Allí los estudiantes ingresaban en carreras como Electrónica, Aeronáutica, Mecánica Naval, Operación Técnica de Radio, Meteorología, Oceanografía. A partir del golpe de Estado de 1976, funcionó paralelamente como uno de los centros clandestinos de detención, tortura y exterminio más emblemáticos de la última dictadura (EMDH 2011). en las sensaciones genera otro tipo de "agencia", en la que los visitantes son actores, y no meramente espectadores, del dolor. Estos autores también afirman que el diseño de monumentos contemporáneo provoca nuevas experiencias que trascienden lo puramente visual (Stevens y Franck 2016:130). Tomando como referencia esta cuarta idea del texto reseñado, vale la pena cuestionar, entonces, el uso del testimonio en el Lugar de la Memoria, la Tolerancia y la Inclusión Social (Lum), del Ministerio de Cultura del Perú, donde pantallas de escala humana presentan frontalmente, y a viva voz, a quienes vivieron afectaciones por hechos violentos en este país; asimismo, con un recurso similar, quizá más austero, en el Musée et Centre de Documentation Mémorial de la Shoah (Museo y Centro de Documentación Memorial de la Shoah), en París (Francia), víctimas francesas del Holocausto relatan experiencias de supervivencia durante la ocupación nazi. Desde mi perspectiva, en estos dos casos, el testimonio opera museográficamente para brindar información de manera cercana y emotiva; sin embargo, no transforma el papel del visitante, al que mantiene estático, como una grabadora portátil.

Los monumentos ofrecen un foro público para que la gente exprese sus puntos de vista acerca del suceso conmemorado, o también para que reflexione sobre su significado político, social y personal (Stevens y Franck 2016:100). Al respecto, Stevens y Franck (2016:32) citan, entre otros, el Memorial a las Víctimas de la Violencia en la Ciudad de México (México), donde las personas pueden escribir y dibujar sobre las superficies libres $y$, con ello, generar diálogos en silencio con algunas de las citas previamente inscritas por la curaduría. La necesidad de dejar mensajes y de escribir se explora intensamente en algunos museos, como el Anne Frank Museum (Museo de Ana Frank), en Ámsterdam (Países Bajos) — visitado en 2016 por quien suscribe-, donde, después de 
la casa y el anexo, se permite escribir digitalmente, en las paredes de la sala final, mensajes que luego se exhiben en pantallas para que los lean otras personas. También el Jüdisches Museum Berlin (Museo Judío de Berlín, Alemania), que analicé personalmente el año pasado, invita a sus visitantes, hacia el final de la exposición permanente, a dejar sobre una gran pared notas escritas en pequeños papeles autoadhesivos, a manera de un libro de visitas. Según Stevens y Franck (2016:32), al dejar tributos ya sean mensajes o dibujos-y estudiar los que otros han dejado, el público participa con los monumentos de una forma personal e íntima, además de que enriquece la experiencia de otros visitantes por razón de que ven cómo el lugar sigue jugando un rol activo en la conmemoración.

La visita a un monumento conmemorativo y a un museo de memoria se debe plantear, evidentemente, de manera diferente de como se plantea un libro de historia, Ileno de fechas y relatos, ya que la experiencia corporal, que implica un esfuerzo mental, emocional y físico de transitar en el espacio - un parque, un edificio o un lugar de memoria, como el Auschwitz-Birkenau Miejsce Pamięci i Muzeum (Memorial y Museo de Auschwitz-Birkenau), en Polonia-, permite un acercamiento directo $y$ un proceso proactivo de exploración e interpretación que integra a los demás visitantes, la arquitectura, el paisaje y aun los objetos que acompañan la curaduría, ya se trate de fotografías, artefactos, elementos personales u obras de arte. Consecuentemente, una función pedagógica y una función conmemorativa se funden en la significación que el cuerpo humano, en un lugar de memoria, le otorga al acto de recordar. Stevens y Franck (2016:226) comentan que los memoriales, efectivamente, pueden enseñar historia, pero de una forma diferente, apelando a la experiencia para lograr un entendimiento profundo y emotivo. La combinación de una participación corporal y cognitiva con un monumento $-\mathrm{y}$ un mu- seo- puede tener un impacto intenso, e incluso psicológico, en los visitantes.

La quinta idea que los autores de Memorials as Spaces of Engagement: Design, Use and Meaning proponen trae a colación la relación del monumento con ese otro cuerpo geográfico de mayor escala: la ciudad (Stevens y Franck 2016:14). ¿De qué formas una ciudad se integra a un monumento o un museo de memoria afecta la vida urbana? Cada vez con mayor planeación plazas, parques y alamedas anexionan monumentos y museos para recobrar historias y memorias recientes, como en el caso del Eje de la Paz y la Memoria, en Bogotá (AMB 2014), proyecto urbano que inscribe al ya construido Centro de Memoria, Paz y Reconciliación (CMPyR) y al futuro Museo Nacional de la Memoria de Colombia como elementos primordiales para promover la recuperación de patrimonio (esculturas y cementerios), así como para renovar y resignificar segmentos de ciudad. Esta activación de rutas simbólicas o "complejos memoriales" (Stevens y Franck 2016:14) se puede ver, por mencionar otro caso, en el Gedenkstätte Berliner Mauer (Memorial del Muro de Berlín, Alemania), que incluye un centro de documentación; ahí, en medio de nuevos edificios y por más de $1 \mathrm{~km}$, se integran lugares de memoria, restos históricos, esculturas, instalaciones y apuestas museológicas que ofrecen información a los transeúntes (Stevens y Franck 2016:45). "Los 'sitios históricos' que son testimonios materiales [...] contribuyen al conocimiento de los hechos y funcionan como denuncia, prueba y evidencia de lo ocurrido. [...] no hay duda que el sitio 'habla' haciendo vibrar la emoción e interrogando el pensamiento" (Pastoriza 2005:90). Por mi parte, complementaría esta cita con el hecho de que, en mi experiencia, nuestra presencia corporal y participativa añade múltiples significados a estos espacios conmemorativos.

Y bien, ¿por qué son paradigmáticos el Vietnam Veterans Memo- rial (Monumento a los Veteranos de Vietnam, Washington, EUA), el NAMES Project (Proyecto NAMES) para el AIDS Memorial Quilt (Edredón Conmemorativo sobre el SIDA, Washington, EUA) y el Denkmal für die Ermordeten Juden Europas (Monumento a los Judíos de Europa Asesinados, Berlín, Alemania)? Aunque a lo largo del libro Stevens y Franck (2016) presentan diversos ejemplos de varias latitudes que van reforzando las ideas ordenadas temáticamente en tres secciones (Memorials as Objects and Spaces; Memorials as Used and Understood; Memorials as Responsibilities), estos tres monumentos siempre tienen consonancia con las cinco ideas fundamentales que se han reseñado: el cambio de la glorificación del vencedor (un héroe masculino, heterosexual, blanco y de élite social) a la conmemoración del sufrimiento por razones de conflicto, origen étnico o enfermedad; la invitación a nuevos usos y apropiaciones que van más allá de una experiencia visual para transformar los monumentos en oportunidades escénicas donde puede haber carcajadas, lamentaciones y cuestionamientos; la activación de formas de participación que hacen relevantes sucesos históricos acontecidos décadas atrás; el hecho de que los nuevos diseños de monumentos integran experiencias que, en suma, rebasan la estrictamente visual, y, por último, nuevas formas de relación con la ciudad que invocan planteamientos urbanos integradores.

Escapan de esta reseña museos y lugares de memoria en Montevideo (Uruguay), Asunción (Paraguay), São Paulo (Brasil) o Santo Domingo (República Dominicana), pero, precisamente, por esta proliferación de esfuerzos alrededor de la memoria en América Latina durante las últimas décadas es oportuno proponer una reflexión que tome en cuenta las tesis que exponen Stevens y Franck (2016), para trasladarlas de los monumentos conmemorativos a los museos de memoria. Si bien el libro disocia estos dos dispositivos, indu- 
dablemente se encuentran ecos que, lejos de resolver preguntas, dan origen a muchas otras. ${ }^{4}$ ¿Cuántas veces es necesario recordar el horror? ¿De qué formas podemos, por medio de los monumentos y los museos de memoria, construir empatía y reconciliación? En medio siglo, ¿cuáles serán los impactos de estos monumentos y museos, cuando, en un pensamiento esperanzador, ya habremos superado la violencia?

Memorials as Spaces of Engagement: Design, Use and Meaning resultará muy atractivo para arquitectos, urbanistas, museólogos e interesados en estudiar contenidos sobre memoria, patrimonio y duelo. Este libro, en todo caso, es un ejemplo fehaciente de que el patrimonio y la memoria tienen un futuro que recordar.

\section{Referencias}

AMB

2014 "Decreto 632 de 2014 por el cual se adopta el Proyecto de Diseño Urbano Eje de la Paz y la Memoria, que integra diferentes Conjuntos Monumentales de Espacio Público en la Ciudad de Bogotá, D. C., y se dictan otras disposiciones", Alcaldía Mayor de Bogotá (AMB), Registro Distrital 5503, 31 de diciembre de 2014, documento electrónico disponible en [http://www. alcaldiabogota.gov.co/sisjur/normas/ Norma1.jsp?i=60318], consultado el 28 de diciembre de 2016.

Bergdoll, Barry

2011 "Un marco para reescenificar la memoria", en V.V. A.A., Museo Me-

\footnotetext{
${ }^{4}$ Cabe citar dos noticias recientes sobre la función de los memoriales y los museos conmemorativos en el actual clima político internacional: la primera, del United States Holocaust Memorial Museum (Museo Estadounidense Conmemorativo del Holocausto), que condenó la retórica de odio en la Conferencia del Nacionalismo Blanco llevada a cabo en noviembre de 2016, luego de la elección de Donald Trump (USHMM 2016); la segunda, una reflexión de Jonathan Jones (2016) en el periódico británico The Guardian que, precisamente, relaciona el memorial de Peter Eisenman, en Berlín, y el de Rachel Whiteread, en Viena, con el "retorno de la derecha en Europa".
}

moria y Tolerancia, Arditti + RDT Arquitectos, México, Arquine, 21-29.

$\mathrm{BCNCH}$

1990 “Decreto Supremo núm. 355.

Crea Comisión de Verdad y Reconciliación", Diario Oficial de la Federación de Chile, Biblioteca del Congreso Nacional de Chile (BCNCH), Santiago, Ministerio del Interior y Seguridad Pública de Chile, 9 de mayo, documento electrónico disponible en [http://www. leychile.cl/Navegar?idNorma $=12618$ \&idVersion=1992-07-08], consultado el 28 de diciembre de 2016.

Brodsky, Ricardo

2011 "Un museo vivo para la memoria de Chile", en V.V. A.A., Catálogo del Museo de la Memoria y Derechos Humanos, Santiago, Midia, 9-12.

Carter, Jennifer

2015 "The Museology of Human Rights", en Karen Busby, Adam Muller y Andrew Woolford, The Idea of a Human Rights Museum, Winnipeg, University of Manitoba Press, 208-226.

\section{$\mathrm{CNMH}$}

2015 Tocó cantar. Travesía contra el olvido, Colombia, Museo Nacional de la Memoria, Centro Nacional de Memoria Histórica, documento electrónico [archivos de audio] disponible en [http://www.centrodememoriahistorica.gov.co/discografia-toco-cantar], consultado el 28 de diciembre de 2016. 2016 ¿Qué es el Centro Nacional de Memoria Histórica?, documento electrónico [página web] disponible en [http://www.centrodememoriahistorica.gov.co/somos-cnmh/que-es-el-centro-nacional-de-memoria-historica], consultado el 16 de enero de 2017.

\section{CRC}

2011 "Ley 1448 por la cual se dictan medidas de atención, asistencia y reparación integral a las víctimas del conflicto armado interno y se dictan otras disposiciones", Bogotá, Congreso de la República de Colombia (CRC), Diario Oficial 48096, 10 de junio, documento electrónico disponible en [http://www. alcaldiabogota.gov.co/sisjur/normas/ Norma1.jsp?i=43043], consultado el 28 de diciembre de 2016.

Crawley, Greer

2012 "Staging Exhibitions: Atmospheres of Imagination", en Suzanne
MacLeod, Laura Hourston y Jonathan Hale, Museum Making: Narratives, Architectures, Exhibitions, Londres y Nueva York, Routledge, 12-20. $\mathrm{EMDH}$

2011a "Los orígenes del predio de la ESMA", Espacio, Memoria y Derechos Humanos (ex ESMA), documento electrónico [página web] disponible en [http://www.espaciomemoria.ar/ origenes.php], consultado el 28 de diciembre de 2016.

2011b "El plan sistemático de represión ilegal", Espacio, Memoria y Derechos Humanos (ex ESMA), documento electrónico [página web] disponible en [http://www.espaciomemoria.ar/dictadura.php\#], consultado el 28 de diciembre de 2016.

FMMJE

2010 "The Information Centre-The Exhibition", en V.V. A.A., Memorial to the Murdered Jews of Europe. Guide to the Information Centre, Kienberg, Foundation Memorial to the Murdered Jews of Europe (FMMJ), F\&W Mediencenter, 14-53.

\section{$\mathrm{GMH}$}

2013 "Resistir reconstruyendo memoria: las iniciativas de memoria", en Informe ¡Basta ya! Colombia: Memoria de guerra y dignidad, Bogotá, Grupo de Memoria Histórica (GMH), Imprenta Nacional, 387-395.

Hein, Hilde

2010 "Looking at Museums from a Feminist Perspective", en Amy K. Levin (ed.), Gender, Sexuality, and Museums, Londres / Nueva York, Routledge, 53-64.

Jelin, Elizabeth

2003 Monumentos, memoriales y marcas territoriales, Madrid, Siglo XXI.

Jones, Jonathan

2016 "War Memorials Have FailedWe Have Forgotten the Chaos of Fascism" [The Guardian], documento electrónico disponible en [https:// www.theguardian.com/artanddesign/ jonathanjonesblog/2016/dec/09/warmemorials-have-failed-peter-eisenman-holocaust], consultado el 29 de diciembre de 2016.

MacLeod, Suzanne, Laura Hourston y Jonathan Hale

2012 "Introduction: Museum Making. 
The Place of Narrative", en S. MacLeod, L. Hourston y J. Hale (eds.), Museum Making: Narratives, Architectures, Exhibitions, Londres / Nueva York, Routledge, XIX-XXIII.

$\mathrm{MMDH}$

2011 Música x Memoria, Santiago, Museo de la Memoria y los Derechos Humanos.

2017 Sobre el Museo. Historia, documento electrónico [página web], Museo de la Memoria y los Derechos Humanos, disponible en [http://ww3.museodelamemoria.cl/sobre-elmuseo/], consultado el 16 de enero de 2017.

Pastoriza, Lila

2005 "La memoria como política pública: los ejes de la discusión", en Marcelo Brodsky (ed.), Memoria en construcción: el debate sobre la ESMA, Buenos Aires, La Marca Editora, 8594.

PDM

2014 "Sobre el Parque de la Memoria", Monumento a las víctimas del terrorismo de Estado Parque de la Memoria, Buenos Aires, Parque de la Memoria (PDM), documento electrónico disponible en [http://parquedelamemoria.org.ar/sobre-el-parque-de-la-memoria], consultado el 27 de diciembre de 2016.

Pino, Ponciano del y José Carlos Agüero

2014 Cada uno, un lugar de memoria. Fundamentos conceptuales del Lugar de la Memoria, la Tolerancia y la Inclusión Social, Lima, Lum.

Stevens, Quentin y Karen A. Franck

2016 Memorials as Spaces of Engagement: Design, Use and Meaning, Londres / Nueva York, Routledge.

USHMM

2016 "Museum Condemns White Nationalist Conference Rhetoric", United States Holocaust Memorial Museum (USHMM), documento electrónico disponible en [https://www. ushmm.org/information/press/press-releases/museum-condemns-white-nationalist-conference-rhetoric], consultado el 29 de diciembre de 2016.

Williams, Paul

2007 Memorial Museums: The Global Rush to Commemorate Atrocities, Oxford / Nueva York, Berg Publishers.

\section{Síntesis curricular del/los autor/es}

\section{Michael Andrés Forero Parra}

School of Museum Studies, University of Leicester (UoL, Reino Unido) arkforero@yahoo.com

Arquitecto con estudios complementarios en arte (Universidad de los Andes [Uniandes], Colombia) y magíster con grado meritorio en museología (University of Leicester [UoL], Reino Unido). Su experiencia en museos abarca trabajo en diversas áreas: educativas, con los museos Nacional de Colombia (MNC, Colombia) y de Arte Moderno de Bogotá (Mambo, Colombia) y la Fundación Amigos de las Colecciones de Arte del Banco de la República; curatoriales, con la Wolverhampton Art Gallery (WAG, Inglaterra) y el Ministerio de Cultura de Colombia para la participación del país en el Smithsonian Folklife Festival (Smithsonian Institution, Estados Unidos de América), y de museografía, con el diseño permanente de la Casa Museo Antonio Nariño de Villa de Leyva (Colombia). Miembro fundador del Museo Q (Colombia), proyecto museológico sobre memorias de personas lesbianas, gays, transgénero y bisexuales (LGTB), actualmente es responsable de la infraestructura para el próximo Museo Nacional de la Memoria y Archivo de los Derechos Humanos de Colombia. Miembro del Consejo Internacional de Museos (International Council of Museums [ICOM], Colombia).

Postulado/Submited 25.07.2016

Aceptado/Accepted 06.01.2017

Publicado/Published 31.01.2017

Intervención, Revista Internacional de Conservación, Restauración y Museología, año 8, número 15, enero-junio de 2017, se terminó de publicar electrónicamente el 31 de enero de 2017, Ciudad de México. 